\title{
ÉTUDE DU TÉGUMENT DES GRAINES DU GENRE GENISTA L. (FABACEAE) AU MAROC
}

\author{
Omar AZZIOUI \& Abbas ES-SGAOURI
}

RÉSUMÉ. Étude du tégument des graines du genre Genista L. (Fabaceae) au Maroc. Le tégument externe des graines de 19 espèces appartenant à sept sections différentes du genre Genista L. au Maroc est étudiée au microscope électronique à balayage. Sept espèces endémiques du Maroc sont étudiées pour la première fois. Cinq d'entre elles, présentent une structure plissée au sens large, et deux montrent des structures plus élaborées. Aucun type d'ornementation n'est spécifique à l'une des sept sections étudiées. La présence de deux structures différentes, au sein d'une même espèce, témoigne de la grande instabilité de ce caractère, au sein d'une même section. Le caractère "tégument de la graine" serait à écarter dans la systématique du genre Genista.

Mots clés. Fabaceae, Genista, M.E.B., graine, micromorphologie, Maroc.

ABSTRACT. Seed surface structures in Genista L. (Fabaceae) in Morocco. The seed coat ornamentation was studied in 19 species of genus Genista L. in Morocco by scanning electron microscopy. Seven endemic species are studied for the first time. Five for them have shown a folded pattern (sensu lato), and two a more complex one. The presence of two different pattern in the same species emphasize the great instability of this character inside a same section. Testa patterns seem taxonomically not significant within the genus Genista.

Key words: Fabaceae, Genista, S.E.M., seeds, micromorphology, Morocco.

\section{INTRODUCTION}

L'aire de distribution du genre Genista est circum-Méditerranée. Au Maroc, il présente une large répartition. Les espèces du genre sont présentes dans les basses et moyennes montagnes (Rif, Moyen Atlas, Haut Atlas, Anti Atlas et Monts du Maroc oriental), ainsi que dans les plaines et plateaux du Maroc atlantique et oriental, et au Maroc saharien. Les taxons sont donc soumis à des bioclimats très différents depuis l'aride jusqu'à l'humide.

Le genre Genista a toujours posé des problèmes en systématique, ceci est vraisemblablement du en partie aux affinités morphologiques entre les espèces de ce genre et celles de Cytisus L. Depuis les travaux de Spach (1844-45) consacrés à la révision du genre Genista et à sa délimitation infragénérique, plusieurs travaux de 
systématique, basés sur des études morphologiques ont été menées sur le genre Genista, sur des caractères intéressant pour la systématique de ce groupe et sur la morphologie de la graine et notamment le tégument de la graine (Rothmaler, 1941; Vicioso, 1953; Gibbs, 1966; Gibbs et Dingwall, 1971; Polhill, 1976; Raynaud, 1979; Bisby, 1981; Maire, 1987). Ce caractère a été bien étudié chez les Genisteae ce qui a permis d'affiner la classification de plusieurs espèces d'Ulex L. (Godeau, 1977) et grâce aux différences de structures trouvées, d'aboutir à des conclusions taxinomiques à des niveaux essentiellement générique ou spécifique chez les Vicieae (Lersten, 1979) ainsi que chez les Papilionaceae (Lersten, 1981; Saint-Martin, 1982, 1986), où plusieurs structures tégumentaires furent décrites, et permirent ainsi leurs utilisation pour l'ébauche d'hypothèse évolutive pour ce caractère. Cette étude a pour but d'étudier le tégument des graines des taxons marocains du genre Genista dont certains sont endémiques et étudiés pour la première fois, et de discuter sa valeur en tant que caractère taxinomique, en relation avec les hypothèses phylogéniques sur les structures tégumentaires chez les Papilionaceae, proposées par Saint-Martin (1986).

\section{MATÉRIEL ET MÉTHODES}

Parmi les 35 taxons que compte le genre Genista au Maroc (Greuter, Burdet \& Long, 1989), nous en avons étudié 19. Ces derniers se répartissent sur les sept sections qui constituent le genre au Maroc. La section Spartiö̈des Spach comprend deux espèces étudiées; G. florida L. et G. pseudopilosa Coss. G. florida est une nanophanérophyte du Haut et Moyen Atlas répandue dans des régions à bioclimat semiaride à hiver froid et subhumide (Haut Atlas, Tizi-n-Tichka, Lippert, 07/07/1989, RAB 62108), G. pseudopilosa est une chaméphyte, endémique du Maroc, de la péninsule Ibérique et de l'Algérie, présente dans le Haut et Moyen Atlas en bioclimat semi-aride à hiver froid, subhumide et humide (Azrou, Jbel Hebbri, Maire, 26/07/1921, RAB 62109). Chez la section Scorpiö̈des Spach, une espèce et deux sous-espèces furent étudiées; $G$. ifniensis A. Caball., qui est une nanophanérophyte, endémique du Maroc, localisée dans l'Anti Atlas, le Haut Atlas, le Maroc atlantique moyen en bioclimat semi-aride et semi-aride à hiver doux (Anti Atlas, Anezi, Azzioui, 29/04/1997), G. scorpius subsp. intermedia J.Emb. \& Maire, plante rare, nanophanérophyte, endémique du Maroc, localisée au Moyen Atlas en bioclimat semi-aride à hiver froid et subhumide (Moyen Atlas oriental, Bechchine, Fennane, 14/05/ 1981, RAB 60925) et G. scorpius subsp. myriantha (Ball) Maire, nanophanérophyte, endémique du Maroc, présente dans le Maroc saharien, les plaines et plateaux du Maroc oriental, le Haut Atlas et l'Anti Atlas en bioclimat aride, semi-aride à hiver froid et subhumide (Haut Atlas, Enzel, Maire, 04/07/ 1924, RAB 62113). Nous avons étudié trois représentants de la section Teline (Medik.) Polhill; G. monspessulana (L.) L. Johnson, nanophanérophyte, présente dans le Maroc atlantique nord, le Rif, en bioclimat subhumide et humide (Tanger, Forêt diplomatique, Sauvage, 24/06/1947, RAB 62111); G. osmariensis Coss., plante rare, chaméphyte, endémique du Maroc, présente dans le Rif en bioclimat humide (Rif sud-ouest, Jbel Kelti, Missaoui, Quaisse, Selod et Veilex, 19/06/ 1966, RAB 24299) et G. segonnei (Maire) P. P.E.Gibbs, plante très rare, chaméphyte, endémique du Maroc, localisée dans l'Anti Atlas (Kest et monts d'Ifni) en bioclimat subhumide (Anti Atlas, Jbel Imzi, Azzioui, 01/ 05/1997). Chez la section Phyllobotrys Spach, nous avons étudié une seule sous-espèce; $G$. anglica subsp. ancistrocarpa (Spach) Maire, plante très rare, nanophanérophyte, endémique de la péninsule Ibérique et du Maroc, localisée dans le Maroc atlantique nord en bioclimat 
subhumide et humide (Forêt du Gharb, Métro et Sauvage, 09/05/1946, RAB 62106). Cinq représentants de la section Voglera (P.Gaertn., B.Mey. \& Schreb) Spach ont été étudié; $G$. hirsuta subsp. erioclada (Spach) C. Raynaud, nanophanérophyte, endémique du Maroc et de l'Algérie, présente dans les plaines et plateaux du Maroc oriental, les monts du Maroc oriental, le littoral de la méditerranée, le Maroc atlantique nord et le Rif, dans un bioclimat aride, semi-aride à hiver doux, subhumide (Rif occidental, Koudia Tassagout, Sauvage, 09/ 07/1961, RAB 6949); G. tournefortii Spach, chaméphyte, endémique du Maroc et de la péninsule Ibérique, présente dans le Moyen Atlas et le Rif sous un bioclimat humide (Moyen Atlas, poste de la côte 1565, Emberger, 18/06/ 1927, RAB 62115); G. triacanthos Brot. plante rare, nanophanérophyte, endémique du Maroc et de la péninsule Ibérique, localisée dans le Rif sous un bioclimat subhumide, humide (Cap Spartel, Jbel Kébir, Azzioui, 28/06/1997); G. tricuspidata Desf. nanophanérophyte, présente dans le Haut Atlas, le Rif, le Maroc atlantique moyen, le Maroc atlantique nord, le littoral de la méditerranée, en bioclimat aride, semi-aride à hiver doux et subhumide (Agadir, Cap Ghir, Azzioui, 29/04/1997) et G. tridens (Cav.) DC., plante rare, nanophanérophyte, endémique du Maroc et de la péninsule Ibérique, localisée dans le Rif en bioclimat subhumide et humide (Cap Spartel, Jbel Kébir, Azzioui, 28/06/1997). Nous avons étudié trois représentants de la section Cephalospartum Spach; G. cephalantha subsp. demnatensis (Murb.) C. Raynaud, plante rare, nanophanérophyte, endémique du Maroc, présente dans le Haut Atlas, les monts du Maroc oriental, le Rif et le littoral de la méditerranée en bioclimat semi-aride à hiver doux et subhumide (Haut Atlas, Toufrite, Podlech, 06/ 07/1989, RAB 62107); G. clavata Poir., plante rare, nanophanérophyte, endémique du Maroc, localisée dans le Maroc atlantique nord et le Rif en bioclimat subhumide (Gharb, Arbaoua,
Gattefossé, 08/05/1959, RAB 5019) et $G$. umbellata (L'Hér.) Poir., plante très rare, nanophanérophyte, endémique du Maroc de la péninsule Ibérique et de l'Algérie présente dans le littoral de la méditerranée (Nkor Triffa) en bioclimat semi-aride à hiver doux (Hidum, Sennen et Mauricio, 03/06/1934, RAB 62116). Enfin nous avons étudié deux représentants de la section Spartocarpus Spach; G. saharae Coss. \& Dur., plante rare, nanophanérophyte, endémique du Maroc et de l'Algérie, présente dans le Maroc saharien en bioclimat aride et saharien (Confluent Ziz-Rheris, Raymond, 221 05/1952, RAB 62112) et G. spartioïdes subsp. pseudoretamoïdes Maire (Maire, 1949) nanophanérophyte, endémique du Maroc et de l'Algérie, présente dans le Moyen Atlas, le Rif, les plaines et plateaux du Maroc oriental, le Maroc atlantique nord, les monts du Maroc oriental et le littoral de la méditerranée, en bioclimat aride, semi-aride à hiver doux, semiaride à hiver froid et subhumide (Jbel Kerker, Sennen et Mauricio, 14/06/1933, RAB 62114). Les subdivisions biogéographiques utilisées sont selon Fennane \& Mathez (1992). Le caractère «rare et très rare» a été utilisé selon la définition citée par Fennane \& Ibn Tattou (1998).

Les graines à maturité ont été prélevées sur les plantes vivantes préalablement récoltées dans leur milieu naturel. Pour des raisons matérielles, nous avons completé nos prélevements par des échantillons d'herbier (RAB). Les graines sont montées sur des plots spéciaux, métallisées par pulvérisation OrCarbone et observées au microscope électronique à balayage. Le MEB utilisé est du type Jeol, JSM-T330.

L'observation au MEB a permis de visualiser et définir les structures de la surface tégumentaire des graines. Il existe différentes terminologies pour décrire les téguments séminaux: nous avons suivi la terminologie proposée par Lersten (1981) et Saint-Martin (1986). 


\section{RÉSULTATS ET DISCUSSION}

L'examen des photographies prises au MEB, nous a permis de noter les différentes structures suivantes:

-Une structure microplissée, où la surface cellulaire est recouverte de microplis plus ou moins enchevêtrés. Les plis sont moins enchevêtrés chez $G$. clavata (fig. 1) que chez G. ifniensis, G. triacanthos, G. tricuspidata et G. tridens, (figs. 2, 3, 4, 5).

-Une structure en plis rugulés, avec des plis irréguliers, mais avec un relief plus accentué par rapport aux microplis. On retrouve cette structure chez G. florida, G. osmariensis et $G$. saharae, (figs. 6, 7, 8).

-Une structure en réseau, le bord de la cellule étant en relief, l'ensemble donne l'idée d'un réseau, cette structure est propre à $G$. tournefortii, (fig. 9).

-Une structure multiréticulée, les bords cellulaires sont en relief et la surface cellulaire est recouverte de microplis. Cette structure caractérise, G. anglica subsp. ancistrocarpa, $G$. cephalantha subsp. demnatensis, $G$. monspessulana et G. segonnei, (figs. 10, 11, $12,13)$.

-Une structure en dépression plissée, chez $G$. scorpius subsp. intermedia où la surface cellulaire se creuse d'un orifice étroit vers lequel convergent les plis rayonnants provenant du contour cellulaire, (fig. 14).

-Une structure en réseau creusé, dans le cas où la surface cellulaire se creuse d'un orifice plus ou moins large, les bords cellulaires devenant plus étroit. L'orifice est nettement moins large chez $G$. hirsuta subsp. erioclada et $G$. pseudopilosa (figs. 15, 16) que chez G. scorpius subsp. myriantha, G. spartioïdes subsp. pseudoretamoïdes et $G$. umbellata qui montrent bien cette structure, (figs. 17, 18, 19).

Nous notons que tous les types décrits sont représentés chez les taxons du Maroc. Chez les endémiques, à l'exception de $G$. scorpius subsp. myriantha qui présente une structure en réseau creusé et de G. scorpius subsp. intermedia, qui montre une structure en dépression plissée, les autres endémiques du Maroc (G. cephalantha subsp. demnatensis, $G$. clavata, $G$. ifniensis, G. osmariensis et $G$. segonnei) ont une structure plissée au sens large (microplissée, en plis rugulée, en réseau ou multiréticulée). Chez les taxons non endémiques on observe à la fois des structures plissées au sens large ( $G$. anglica subsp. ancistrocarpa, G. florida, G. monspessulana, $G$. saharae, $G$. tournefortii, G. triacanthos, $G$. tricuspidata et $G$. tridens) et des structures en réseau creusé (G. pseudopilosa, G. hirsuta subsp. erioclada, G. umbellata et $G$. spartioides subsp. pseudoretamoïdes): 13 taxons parmi les 19 étudiés présentent donc une structure plissée au sens large. Ceci rejoint en partie les résultats et les conclusions de Saint-Martin (1986), qui avait observé essentiellement des structures microplissées et plissées chez les taxons de Genista.

Par ailleurs, aucun type d'ornementation n'est spécifique à une section, comme par exemple la section Voglera qui présente les structures microplissées, en réseau et en réseau creusé, ou comme la structure en réseau creusé qui se retrouve au niveau de cinq sections différentes. Le caractère "tégument" ne permet pas de discriminer de la même manière que les autres caractères morphologiques (plante inerme ou épineuse; feuille simple ou trifoliée, nombre de faisceaux libéro-ligneux au niveau du point d'insertion des feuilles sur la tige, forme de l'étendard, forme de la gousse, nombre de graines etc.); lesquels ont été utilisés par Gibbs (1966) pour réviser la classification infragénérique, proposée par Spach (1844-45).

En effet, les structures tégumentaires de G. monspessulana, G. osmariensis, et $G$. segonnei sont similaires à celles présentes chez plusieurs espèces de Genista, alors qu'elles ont des caractères morphologiques communs aux taxons du genre Cytisus. Ces trois espèces 

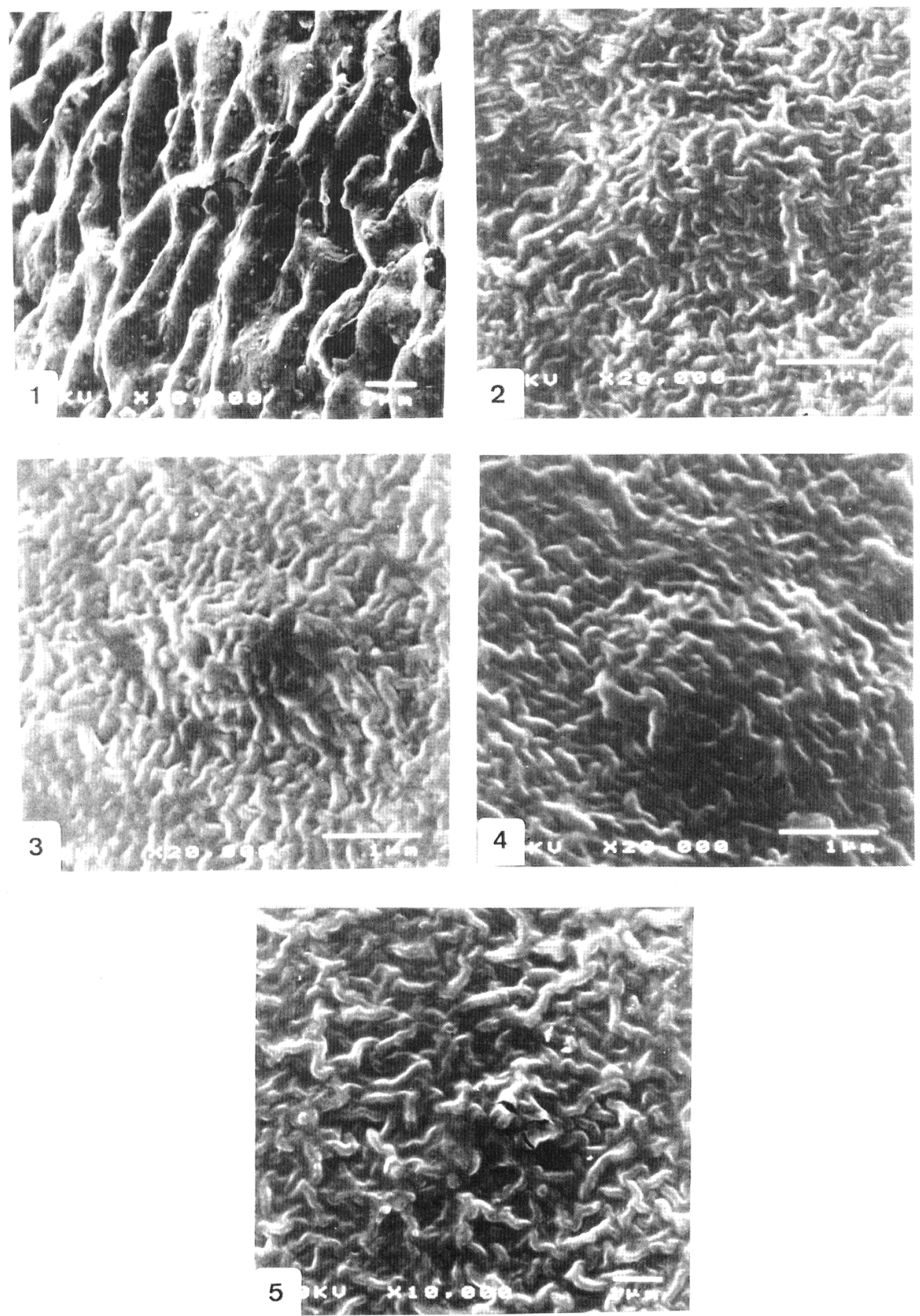

Figures 1 à 5: Structure microplissée; fig. 1: G. clavata, fig. 2: G. ifniensis, fig. 3: G. triacanthos, fig. 4 : G. tricuspidata, fig. 5: G.tridens. 

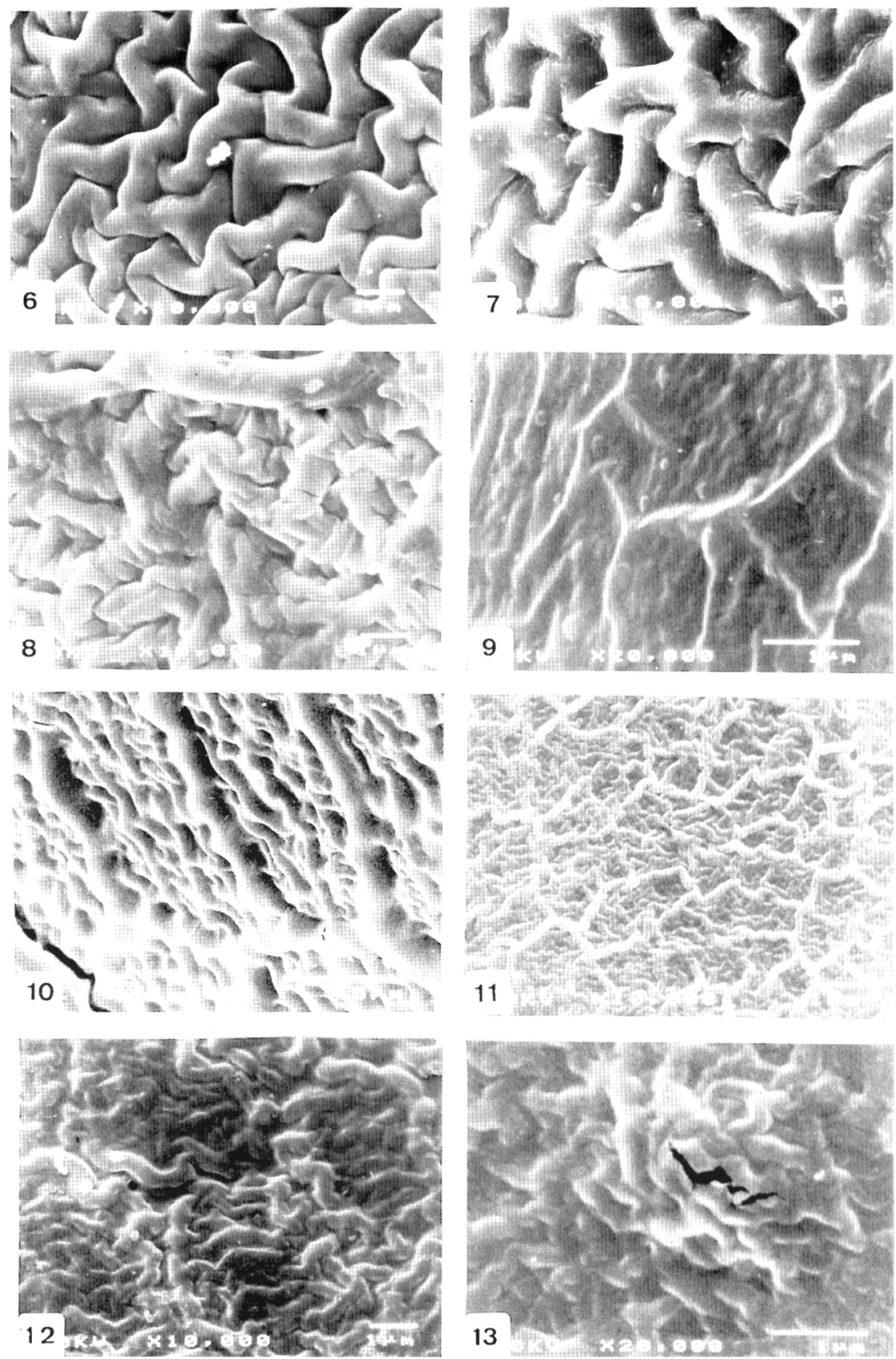

Figures 6 à 8: Structure en plis rugulés; fig. 6: G. florida, fig. 7: G. osmariensis, fig. 8: G. saharae. Fig. 9: Structure en réseau; fig. 9: G. tournefortii. Fig. 10 à 13: Structure mutiréticulée; fig. 10: G. anglica subsp. ancistrocarpa, fig. 11: G. cephalantha subsp. demnatensis, fig. 12: G. monspessulana, fig. 13: $G$. segonnei. 

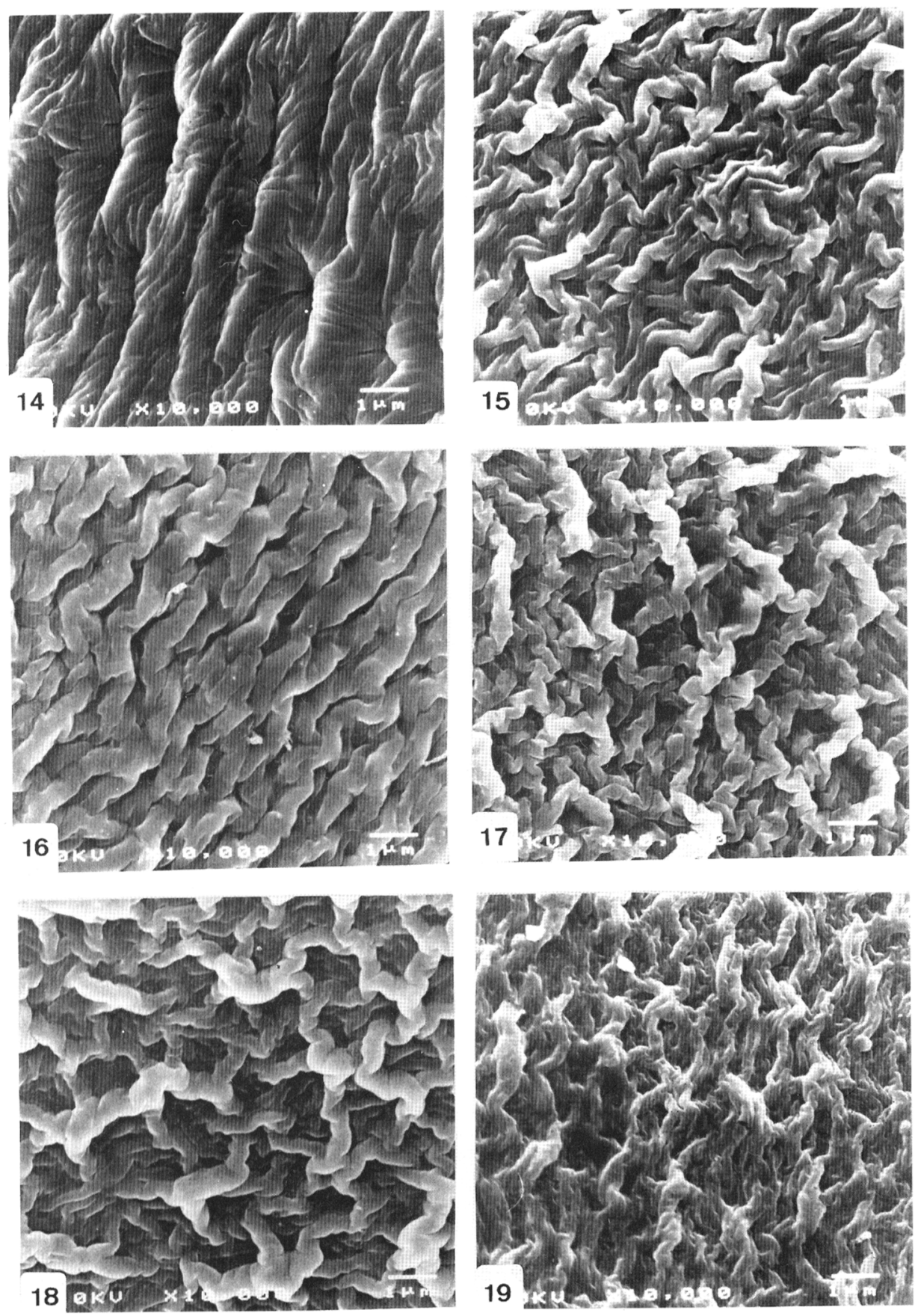

Figure 14: Structure en dépréssion plissée; fig. 14: G. scorpius subsp. intermedia. Fig. 15 à 19: Structure en réseau creusé; fig. 15: G. hirsuta subsp. erioclada, fig. 16: G. pseudopilosa, fig. 17: G. scorpius subsp. myriantha, fig. 18: G. spartioüdes subsp. pseudoretamoïdes, fig. 19: G. umbellata. 
ont pour celà alimenté un conflit systématique qui les place soit, en se basant sur la présence de l'arille et des feuilles trifoliées, dans le genre Cytisus section Teline (Medicus) Benth., soit, en tenant compte entre autres de la lèvre supérieure du calice profondément bifide et des ailes oblongues, dans le genre Teline (Medicus) Gibbs \& Dingwall, soit enfin, en considérant des caractères floraux, dans le genre Genista section Teline (Medicus) Polhill. Cette dernière position systématique a été confortée par les études sérologiques menées par Cristofolini et Chiapella (1984), qui montrent que les espèces de la section Teline ont une réactivité sérologique similaire à celles des autres sections de Genista subgen. Genista. En outre, la présence de deux structures tégumentaires différentes au sein de la même espèce $G$. scorpius témoigne de la grande instabilité de ce caractère au sein d'une même section. Ces résultats seraient un argument pour écarter ce caractère de la systématique ou l'utiliser avec prudence.

Par ailleurs, dans une étude portant sur le tégument externe des graines des Papilionaceae, Saint-Martin (1986) avait noté différentes structures qui lui ont servi de base pour définir l'évolution de ce caractère. C'est ainsi qu'il considère que l'évolution s'est déroulée dans le sens d'une spécialisation de plus en plus poussée de l'ornementation. Les structures microplissées et plissées propres aux Genisteae seraient les plus primitives, et celle en réseau creusé, plus élaborée, observée chez les Coronilleae, serait la plus évoluée. Nos résultats sur les structures tégumentaires n'appuient pas formellement les hypothèses phylogéniques de Saint-Martin (1986). En effet, la structure en réseau que Saint-Martin (1986) signale chez Retama monosperma (L.) Boiss. et Retama sphaerocarpa (L.) Boiss., se retrouve chez $G$. tournefortii. Certains de nos taxons présentent aussi des structures qui se trouvent chez des taxons appartenant à des tribus placées par Polhill (1981) à un niveau plus évolué que les Genisteae. Ainsi, la structure en dépression plissée propre à plusieurs espèces de Loteae, Anthyllis barba-jovis L. (Linné, 1753), Anthyllis cytisoides L., Anthyllis hermanniae L., Anthyllis montana L., Anthyllis tetraphylla L., Dorycnium rectum (L.) Ser., Lotus angustissimus L., L. corniculatus L., L. creticus L., L. parviflorus Desf., L. subbiflorus Lag., L. uliginosus Schkuhr, Securigera securidaca(L.) Deg. \& Dör. se retrouve chez G. scorpius subsp. intermedia. La structure en réseau creusé que Saint-Martin (1986) considère comme étant évoluée, et, qu'il signala chez plusieurs espèces de Coronilleae, Coronilla minima L., C. scorpioides (L.) Koch, C. valentina subsp. glauca (L.) Batt., Hippocrepis comosa L., H. multisiliquosa L., H. unisiliquosa L., se retrouve chez Genista hirsuta subsp. erioclada, G. pseudopiloda, G. scorpius subsp. myriantha, $G$. spartioüdes subsp. pseudoretamoïdes et $G$. umbeliata.

En conclusion, il est difficile dans l'état actuel des connaissances d'approcher les aspects évolutifs des caractères liés à la micromorphologie des graines chez les espèces de Genista. Dans cette perspective, une étude caryologique permettrait d'associer les niveaux de ploïdie éventuels aux caractères séminaux, et pourrait donc nous informer sur l'état évolutif de ces caractères.

REMERCIEMENTS. Nous remerçions vivement les chercheurs du Département de Botanique et d'Ecologie Végétale de l'Institut Scientifique à Rabat dans lequel ce travail a été conçu et réalisé.

\section{BIBLIOGRAPHIE}

BISBY, F. A. -1981-Tribe 32. Genisteae (Adans.) Benth. (1865). In Advances in Legume Systematics, Vol 1 (Polhill, R. M. and Raven, P. H., eds), pp. 409-425. Kew Royal Botanic Gardens, London.

CRISTOFOLINI, G. \& L. FEOLI CHIAPELLA - 
1984- Origin and diversification of «Genisteae» (Fabaceae): a serosystematic purview. Webbia 38: 105-122.

FENNANE, M. \& M. IBN TATTOU -1998- Catalogue des plantes vasculaires rares, menacées ou endémiques du Maroc. Bocconea 8.

FENNANE, M. \& J. MATHEZ -1992- Eléments pour la flore pratique du Maroc. Naturalia Monspel. Sér. Bot. 56: 171-220.

GIBBS, P.E. -1966- A revision of the genus Genista L. - in Notes Royal Bot. Gard., Edinburgh 27 (1):11-99.

GIBBS, P.E. \& I. DINGWALL - 1971- A revision of the genus Teline. Bol. Soc. Broteriana, sér. 2, 45: 269-316.

GIBBS, P.E. -1974- Lagascalia 4: 39.

GODEAU, M. -1977- Observation au microscope électronique à balayage de l'épiderme séminal d'Ulex europaeus L., U.minor Roth., U.gallii Planchon. Bull. Soc. Sci. nat. Ouest France LXXV: 83-89.

GREUTER, W., H.M. BURDET \& G. LONG -1989Med-Checklist, 4. Genève: Editions des Conservatoire et Jardin Botaniques.

LERSTEN, N.R. -1979- A distinctive seed coat patterne in the Vicieae (Papilionoideae; Leguminosae). Proc. Iowa Acad. Sci. 86 (3): 102-104.

LERSTEN, N.R. -1981- Testa topography in Leguminosae, sub-family Papilionoideae. Proc. Iowa Acad. Sci. 88 (4): 180-191.

MAIRE, R. -1987-Flore de l'Afrique du Nord, vol XVI, pp: 123-300. Ed. Lechevalier, Paris.

POLHILL, R.M. -1976- Genisteae (Adans.)Benth. and related tribes (Leguminosae). In Botanical Systematics, vol. 1 (Heywood V.H., ed.), pp. 143-368. London.

POLHILL, R.M. -1981-Delimitation of tribes in the Papilionoideae. P. 191-208. In Polhill, R.M., and P. Raven (eds), Advances in Legume systematics. Vol. 2 of Proceeding of international legume conference, Kew, 1978. Ministry of Agriculture, Fisheries and Food, Richmond, England.

RAYNAUD, C. -1979- Le genre Genista L. au Maroc. Monographie, iconographie, clés de détermination. Naturalia monspeliensia sér. Bot. Fasc. 28 p. 1-52.

ROTHMALER, W. -1941- Revision der Gensteen I: Monographien der Gattungen um Ulex. Bot.
Jahr. 72: 69-116.

SAINT-MARTIN, M. -1982-Biosystématique des Papilonaceae. Ontogénie, phytodermologie, séminologie. Thèse Univ. Paul Sabatier, Toulouse. 306p, 24pl.

SAINT-MARTIN, M. -1986- Micromorphologie tégumentaire des graines de Papilionaceae. Bull. Soc. Bot. Fr., 133, Lettres bot., 1986 (2), 137 153.

SPACH, E. -1844-45- Revision genero Genista.-in Anales des sciences nat., sér. Bot. 2: 237-279; 3: 102-158.

VICIOSO, C. -1953-1955- Genisteas espanolas.-in Inst. for. Inv. exper. Madrid, 67-72.

Aceptado para su publicación en Septiembre de 1999

Direction des auteurs. Département de Biologie, Faculté des Sciences Aïn Chok, Casablanca, Maroc. 